\title{
Dor pélvica crônica e suas repercussões em mulheres assistidas na Atenção Primária
}

\author{
Chronic pelvic pain and its repercussions in women assisted in Primary Care \\ Dolor pélvico crónico y sus repercusiones en mujeres atendidas en Atención Primaria
}

\author{
Ilis Maria Lucas Xavier \\ ORCID: https://orcid.org/0000-0002-5102-3312 \\ Centro Universitário do Maranhão, Brasil \\ E-mail: ilisxavier@hotmail.com \\ Cristina Maria Douat Loyola \\ ORCID: https://orcid.org/0000-0003-2824-6531 \\ Centro Universitário do Maranhão, Brasil \\ E-mail: crisloyola@hotmail.com \\ Rosane Mara Pontes de Oliveira \\ ORCID: https://orcid.org/0000-0002-2183-1423 \\ Universidade Federal do Rio de Janeiro, Brasil \\ E-mail: romapope@gmail.com \\ Maria Giovana Borges Saidel \\ ORCID: https://orcid.org/0000-0002-3259-1760 \\ Universidade Estadual de Campinas, Brasil \\ E-mail: mgsaidel@unicamp.br \\ Flor de Maria Araujo Mendonça \\ ORCID: https://orcid.org/0000-0002-2796-0939 \\ Centro Universitário do Maranhão, Brasil \\ E-mail: floragyhn@gmail.com \\ Marcos Antonio Barbosa Pachêco \\ ORCID: https://orcid.org/0000-0002-3566-5462 \\ Centro Universitário do Maranhão, Brasil \\ E-mail:mmmarco@terra.com.br
}

\begin{abstract}
Resumo
A dor pélvica crônica é um processo doloroso que interfere nas atividades diárias da mulher, necessitando de intervenções clínicas ou cirúrgicas. O objetivo deste estudo é apresentar as repercussões da dor pélvica crônica em mulheres assistidas na atenção primária. Trata-se de um estudo transversal e quantitativo, conduzido com mulheres assistidas em três unidades da atenção primária e em um ambulatório especializado localizados em São Luís, Maranhão, Brasil, no período de maio a outubro de 2018. Utilizou-se um questionário estruturado. Dos resultados, destacam-se as seguintes prevalências sobre o perfil das mulheres: idade de 18 a 23 anos (18\%), ensino médio completo (46\%), desempregadas (43,3\%), com hipertensão arterial e diabetes (23\%), com duração da dor por dias (60\%), escala de dor de 7 a 10 (73\%), influência negativa da dor nos relacionamentos familiares (63\%), com insônia (59\%), ansiedade (78\%) e depressão (32\%). Constatou-se uma predominância de mulheres jovens, com escolaridade média, desempregadas, com padrão de eliminações intestinais disfuncional, com dores persistentes e intensas e dispareunia. A dor pélvica crônica causou prejuízos na vida social, na qualidade de vida e na saúde psicológica das participantes, o que indica a necessidade de intervenções multidisciplinares em saúde.
\end{abstract}

Palavras-chave: Prevalência; Mulheres; Dor crônica.

\begin{abstract}
Chronic pelvic pain is a painful process that interferes with women's daily activities, requiring clinical or surgical interventions. The aim of this study is to present the repercussions of chronic pelvic pain in women assisted in primary care. This is a cross-sectional and quantitative study, conducted with women assisted in three primary care units and one specialized outpatient clinic located in São Luís, Maranhão, Brazil, from May to October 2018. A structured questionnaire was used. From the results, the following prevalence on the profile of women stood out: age from 18 to 23 years (18\%), complete high school (46\%), unemployment (43.3\%), women with arterial hypertension and diabetes (23\%), with pain duration for days $(60 \%)$, pain scale from 7 to $10(73 \%)$, negative influence of pain on family relationships (63\%), with insomnia (59\%), anxiety (78\%) and depression (32\%). There was a predominance of young women, with medium schooling, unemployed, with dysfunctional bowel elimination pattern, with persistent and intense pain and dyspareunia. Chronic pelvic pain has impaired the social life, quality of life and psychological health of the participants, which indicates the need for multidisciplinary health interventions.
\end{abstract}

Keywords: Prevalence; Women; Chronic pain. 


\begin{abstract}
Resumen
El dolor pélvico crónico es un proceso doloroso que interfiere con las actividades diarias de la mujer y requiere intervenciones clínicas o quirúrgicas. El objetivo de este estudio es presentar las repercusiones del dolor pélvico crónico en mujeres asistidas en atención primaria. Se trata de un estudio transversal y cuantitativo, realizado con mujeres atendidas en tres unidades de atención primaria y en un ambulatorio especializado en São Luís, Maranhão, Brasil, de mayo a octubre de 2018. Se utilizó un cuestionario estructurado. De los resultados se destacan las siguientes prevalencias en el perfil de las mujeres: edad de 18 a 23 años (18\%), enseñanza media completa (46\%), desempleadas (43,3\%), con hipertensión arterial y diabetes (23\%), con duración del dolor por días (60\%), escala de dolor de 7 a 10 (73\%), influencia negativa del dolor en las relaciones familiares (63\%), con insomnio (59\%), ansiedad (78\%) y depresión (32\%). Hubo predominio de mujeres jóvenes, con escolaridad media, desempleadas, con patrón de evacuación intestinal disfuncional, con dolor persistente e intenso y dispareunia. El dolor pélvico crónico ha deteriorado la vida social, la calidad de vida y la salud psicológica de las participantes, lo que indica la necesidad de intervenciones de salud multidisciplinarias.

Palabras clave: Prevalencia; Mujeres; Dolor crónico.
\end{abstract}

\title{
1. Introdução
}

A dor pélvica crônica (DPC) compreende uma dor contínua ou intermitente, com duração igual ou superior a seis meses, fora do período menstrual, localizada na região infra abdominal ou pélvica. A vivência deste processo doloroso interfere nas atividades diárias da mulher necessitando então de intervenções clínicas e/ou cirúrgicas. Trata-se de disfunção musculoesquelética relacionada a diversos sintomas pélvicos, dos quais destacam-se: dor pélvica, dispareunia, dor na micção e dificuldades de evacuação (Bercrombrie \& Learman, 2012; Ahangari, 2014). Esta condição atinge entre 5,7 e 26,6\% das mulheres do mundo (Silva et al., 2011) e, no Brasil, a prevalência é de 15,1\% em mulheres em idade fértil (Coelho et al., 2014), e 19\% em mulheres com idade entre 14 e 60 anos (Williams et al., 2004). No que diz respeito a etiologia, não é possível identificar um único fator. Estudos demonstram que em pelo menos metade dos casos existem uma ou mais comorbidades associadas, como a síndrome do intestino irritável, cistite intersticial/síndrome da bexiga dolorosa, endometriose ou aderências pélvicas (Haggerty et al., 2005; Tirlapur et al., 2013). A presença de endometriose concomitantemente com a cistite intersticial não é incomum (Loduca et al., 2014).

Uma parcela importante de mulheres com DPC não apresenta nenhum tipo de alteração ao exame físico e nem no exame de ultrassonografia, em muitos casos inclusive não é possível encontrar nenhum diagnóstico orgânico. Os diversos fatores psicológicos, que constituem a dimensão afetiva da dor, são evidenciados como importante potencial na influência da percepção da dor, podendo influenciar tanto no diagnóstico quanto no tratamento (Loduca \& Samuelian, 2009; Brilhante et al., 2019).

Pacientes que convivem com dores crônicas, em geral, possuem longa história de dor e usualmente sintomas de sofrimento psíquico. Como consequência possível da DPC, é possível haver comprometimento laborativo e perda e/ou redução da capacidade funcional para desempenhar funções cotidianas (Bryant et al., 2016). Mulheres com DPC apresentam prevalências altas relacionadas a sintomas de sofrimento psíquico e transtornos mentais quando comparadas com mulheres sem DPC. Em um ambulatório especializado para DPC mais de 50\% das mulheres apresentavam ansiedade moderada a grave e mais de $25 \%$ apresentavam depressão moderada a grave (Till; As-Sanie \& Schrepf, 2019).

Frente às afirmações apresentadas o objetivo do estudo é apresentar a as repercussões da dor pélvica crônica em mulheres assistidas na atenção primária.

\section{Metodologia}

Trata-se de um transversal e quantitativo sobre a prevalência de dor pélvica crônica (DPC) e suas repercussões em mulheres assistidas na atenção primária. A coleta foi realizada no período de maio a outubro de 2018. Os campos de coleta de dados (três unidades de saúde e um ambulatório especializado) estão localizados em São Luís, um município de grande porte no estado do Maranhão, Brasil. A amostra foi do tipo não probabilística por conveniência sendo incluídas no estudo: mulheres, com 
diagnóstico de DPC, maiores de 18 anos e que estivessem disponíveis e interessadas em participar da pesquisa. Os critérios de exclusão foram: gestantes, pacientes com câncer e com déficit cognitivo, determinado com base na experiência clínica da pesquisadora responsável pela coleta. Para o recrutamento das participantes nos locais de coleta, realizou-se, inicialmente, uma consulta ao sistema de informações do serviço de atenção integral à saúde da mulher do município, permitindo identificar 165 mulheres com DPC.

Para a coleta de dados, utilizou-se um questionário estruturado com dados sociodemográficos (idade, escolaridade, ocupação), clínicos (doenças crônicas, frequência das evacuações), escala numérica de dor e questões objetivas sobre a percepção da DPC (duração das dores, interferência da dor na relação com familiares, compartilhar sobre a dor com terceiros, presença de sintomas relacionados à insônia, ansiedade e depressão) e sobre o atendimento (características da consulta clínica com o profissional médico). A entrevista ocorreu nos locais de atendimento, em sala reservada e foi conduzida pela primeira autora do trabalho, médica, mestranda e com expertise da prática clínica.

Após a realização das entrevistas, foi construído um banco de dados com todas as informações coletadas. Os dados foram inseridos em planilhas do Excel para obtenção de frequências simples e descrição das diferentes variáveis elencadas no instrumento de coleta de dados. Dessa forma, trata-se de uma análise estatística descritiva simples.

Sobre os aspectos éticos, o estudo foi aprovado no Comitê de Ética em Pesquisa do Centro Universitário do Maranhão (UNICEUMA) sob o parecer $n^{\circ}$ 2.612.749. Todas as participantes assinaram o Termo de Consentimento Livre e Esclarecido (TCLE).

\section{Resultados}

A consulta ao sistema de informações permitiu identificar 165 mulheres com DPC. Destas, 150 atenderam aos critérios de seleção e aceitaram participar da pesquisa, sendo, assim, incluídas na amostra final. O ambulatório concentrou o maior número de atendimentos das mulheres que participaram deste estudo (64,7\% versus 35,3\% provenientes das três unidades básicas), o que demonstra a busca dessas mulheres por atendimento especializado.

A partir da Tabela 1 infere-se, sobre a idade das mulheres, uma prevalência maior de mulheres jovens, com idade de 18 a 23 anos (18\%). Ainda é possível observar nos dados sobre grupo etário que há uma diminuição da prevalência de dor pélvica crônica conforme idade mais avançada, e apenas $6 \%$ da amostra encontrava-se no grupo de 53 a 58 anos. A escolaridade das participantes concentrou-se no ensino médio incompleto (46\%) seguida do ensino médio completo (28\%), apenas $11 \%$ da amostra apresentaram nível de escolaridade superior completo. Sobre a ocupação, a maioria das mulheres (43,3\%) estavam desempregadas no momento da consulta. 18\% apresentam diagnóstico concomitante de Hipertensão Arterial Sistêmica e 5\% Diabetes Mellitus. O padrão de funcionamento intestinal chamou a atenção dos pesquisadores, $61,7 \%$ das participantes apresentam frequência de evacuação apenas uma vez na semana.

Nas variáveis que dizem respeito à dor, $60 \%$ das mulheres relataram duração de dias nas crises agudas de DPC, 73\% atingiram de 6 a 10 pontos na escala de expressão da dor nas crises agudas e $44 \%$ sofrem com dispaurenia. Durante as consultas com o profissional médico, 50\% relataram ter sido submetidas à exame físico e 59\% tiveram exames de imagem solicitados.

Nos aspectos comportamentais/emocionais relatados, $63 \%$ das mulheres relataram influência da DPC negativa na relação com familiares, $65 \%$ compartilham a dor com terceiros (amigas e familiares mais próximos), 59\% apresentam insônia, $78 \%$ relatam sentir ansiedade e $32 \%$ depressão. 
Tabela 1 - Perfil de mulheres com dor pélvica crônica assistidas na atenção primária de São Luís, Maranhão, de maio a outubro de 2018.

\begin{tabular}{|c|c|}
\hline Variáveis & $\%$ \\
\hline \multicolumn{2}{|l|}{ Idade } \\
\hline 18 a 23 anos & $18 \%$ \\
\hline 23 a 28 anos & $12 \%$ \\
\hline 28 a 33 anos & $13,4 \%$ \\
\hline 33 a 38 anos & $14 \%$ \\
\hline 38 a 43 anos & $14,7 \%$ \\
\hline 43 a 48 anos & $12,6 \%$ \\
\hline 48 a 53 anos & $9,3 \%$ \\
\hline 53 a 58 anos & $6 \%$ \\
\hline \multicolumn{2}{|l|}{ Escolaridade } \\
\hline Fundamental completo & $15 \%$ \\
\hline Médio incompleto & $46 \%$ \\
\hline Médio completo & $28 \%$ \\
\hline Superior completo & $11 \%$ \\
\hline \multicolumn{2}{|l|}{ Ocupação } \\
\hline Desempregada & $43,3 \%$ \\
\hline Estudante & $10,7 \%$ \\
\hline Técnico(a) de enfermagem & $4,3 \%$ \\
\hline Doméstica & $25,2 \%$ \\
\hline Serviços Gerais & $16,5 \%$ \\
\hline \multicolumn{2}{|l|}{ Doença Crônica } \\
\hline Hipertensão Arterial Sistêmica & $18 \%$ \\
\hline Diabetes Mellitus & $5 \%$ \\
\hline \multicolumn{2}{|l|}{ Frequência de evacuações } \\
\hline Uma vez na semana & $61,7 \%$ \\
\hline A cada 3 dias & $2,7 \%$ \\
\hline Diariamente & $3,3 \%$ \\
\hline Não souberam responder & $2,3 \%$ \\
\hline \multicolumn{2}{|l|}{ Duração das dores } \\
\hline As crises agudas duram dias & $60 \%$ \\
\hline As crises agudas duram horas & $37 \%$ \\
\hline As crises agudas duram meses & $3 \%$ \\
\hline Dispaurenia & $44 \%$ \\
\hline 1 a 5 na escala de expressão da dor nas crises agudas & $28 \%$ \\
\hline 6 a 10 na escala de expressão da dor nas crises agudas & $73 \%$ \\
\hline \multicolumn{2}{|l|}{ Características da consulta clínica com o profissional médico } \\
\hline Submetida a exame físico durante a consulta & $50 \%$ \\
\hline Solicitado exame de imagem & $59 \%$ \\
\hline \multicolumn{2}{|l|}{ Percepção dos aspectos comportamentais/emocionais relatados } \\
\hline A dor influencia negativamente na relação com familiares & $63 \%$ \\
\hline Compartilham a dor com terceiros & $65 \%$ \\
\hline Insônia & $59 \%$ \\
\hline Sintomas de Ansiedade & $78 \%$ \\
\hline Sintomas de Depressão & $32 \%$ \\
\hline
\end{tabular}

Fonte: Dados da pesquisa (2018).

\section{Discussão}

A partir dos dados do presente estudo constatou-se, na amostra de 150 mulheres com DPC, uma predominância de mulheres jovens, com escolaridade média, desempregadas, com padrão de eliminações intestinais disfuncional, com dores persistentes e intensas e dispareunia. A literatura mostra um incremento de pesquisas na temática, porém, estudos em larga escala ainda são predominantes nos países desenvolvidos. Assim, é preciso considerar as mulheres com DPC nos países em desenvolvimento, incluindo o Brasil. 
O Brasil é um país de dimensão continental, por isso, os aspectos relacionados à dor pélvica e as características das mulheres brasileiras podem sofrer variações importantes dependendo da região do país estudada. Estudos com diferentes composições e triangulações de amostras são fundamentais para a ampliação do entendimento sobre DPC e consequentemente aprimoramento da assistência à saúde dessa mulher. Outro ponto importante de enfatizar antes da discussão é que devido à complexa etiologia da DPC, em relação aos benefícios terapêuticos, os melhores resultados são atingidos quando a terapêutica é fundamentada em uma abordagem interdisciplinar (Wozniak, 2016).

O perfil sociodemográfico identificado no presente estudo é característico da população que busca atendimento na atenção primária e pública. Na amostra do presente estudo, 43,3\% das mulheres relataram estar desempregadas, esse dado parece ser significativo quando encontramos na literatura alguns resultados que corroboram com essa informação da atividade ocupacional e níveis de incapacidade funcional. Estudo internacional com amostra de 149 mulheres de um hospital universitário descreveu que os níveis de incapacidade funcional são influenciados por diversos fatores, dos quais destacam-se: preocupações, medos e possíveis explicações incorretas relacionadas à DPC (Roth; Punch \& Bachman, 2011).

Sobre outros dados encontrados na amostra, os aspectos dos hábitos intestinais relatados são fundamentais e devem compor a história clínica dessas mulheres com queixas de DPC. Estudo que avaliou o efeito da constipação funcional em mulheres com e sem DPC concluiu que apesar da constipação funcional não ter um papel no agravamento do dor ela foi significativamente mais prevalente com mulheres com DPC (Singh et al., 2019). Esse estudo corrobora com os resultados encontrados na presente amostra. Outro estudo mais recente, que dentre os seus objetivos comparou disfunções do assoalho pélvico e a constipação intestinal demonstrou que pacientes com constipação apresentam sofrimento significativo incluindo sintomas urinários e prolapso de órgãos pélvico. Contudo, os achados não foram totalmente explicados por dor abdominal (Roth; Punch \& Bachman, 2011).

O exame físico foi realizado, segundo relato das participantes, em $50 \%$ das consultas. Durante as consultas, 59\% das mulheres tiveram exames de imagem solicitados. A literatura enfatiza que os principais métodos diagnósticos empregados são os exames de imagem, dos quais destacam-se a ultrassonografia, a tomografia computadorizada e a ressonância magnética (Wozniak, 2016). A importância destes exames se dá pela possibilidade de identificar a causa da dor crônica, evidenciar patologias sobrepostas ou para descartar certas patologias. Por outro lado, a acurácia destes exames é debatida pois depende de habilidades do examinador (Lin et al., 2018).

Quanto às repercussões da DPC, constatou-se que a DPC causou prejuízos na vida social, na qualidade de vida e na saúde psicológica das participantes. Um estudo recente concluiu que condições psicológicas, como comorbidades, são altamente prevalentes entre pacientes com DPC (Siqueira-Campos et al., 2019). Essas condições estão associadas ao aumento da intensidade da dor e diminuição da qualidade de vida. A compreensão da sobreposição de condições de dor crônica e transtornos mentais é complexa, contudo, parece que existem fatores ambientais, genéticos, inflamatórios e fatores neurobiológicos que aumentam a vulnerabilidade de ambas as condições. Nesse sentido, mulheres com DPC e transtornos mentais são mais bem assistidas por equipes multidisciplinares incorporando os tratamentos farmacológicos e opções não farmacológicas, além de processos de articulação das equipes da atenção primária com as da atenção especializada.

$\mathrm{O}$ transtorno depressivo maior é mais prevalente em mulheres e os sintomas de dor são queixas comuns em pacientes com sintomas depressivos, aproximadamente $65 \%$ dos pacientes com depressão relatam algum tipo de sintoma de dor crônica. Pacientes com depressão e dor crônica apresentam mais limitações funcionais, percepção de piora da qualidade de vida e não adesão ao tratamento quando comparados com pacientes apenas com depressão ou apenas com dor crônica (Bryant et al., 2016).

No caso de pacientes com DPC, a prevalência da depressão varia de 26-52\%, sendo que na população geral este número é de 10\% (Romão et al., 2009). Na amostra estudada, 32\% das participantes relataram percepção de sintomas de depressão, convergindo então com este estudo. Estudos evidenciam, ainda, que pacientes com DPC que têm concomitantemente depressão 
vivenciam a dor de maneira mais intensa e relatam qualidade de vida significativamente mais baixa (Bryant et al., 2016; Yosefm et al., 2016; Modesto \& Bahamondes, 2011).

Uma situação semelhante ocorre com o transtorno de ansiedade generalizada, que também é altamente prevalente entre pacientes com dor crônica. Esta condição é caracterizada por preocupação generalizada ou excessiva que interfere nas atividades diárias sono e concentração. Na ansiedade, assim como na depressão, mulheres correm maior risco de desenvolver transtornos de ansiedade em comparação com os homens.

Entre pacientes com DPC a prevalência de ansiedade varia de 39 a $73 \%$ em comparação com $12 \%$ da prevalência na população geral (Bryant et al., 2016; Romão et al., 2009; Yosefm et al., 2016; Modesto \& Bahamondes, 2011). Na amostra estudada, 78\% das mulheres relataram sintomas de ansiedade, superior à prevalência apresentada, esse achado pode estar relacionado à situação socioeconômica e de vulnerabilidade da amostra estudada quando comparada com estudos de países desenvolvidos. Assim como na depressão, estudos descrevem que a ansiedade e DPC concomitantes também experimentam mais dor e diminuição relatada da qualidade de vida (Yosefm et al., 2016; Modesto \& Bahamondes, 2011). A ansiedade e a depressão usualmente são vistas juntas em pacientes com DPC (Bryant et al., 2016; Yosefm et al., 2016). Contudo estudo recente com amostra de 25 mulheres e aplicação de escalas diversas constatou que os fatores psicossociais que apresentaram maior pontuação média foram a ansiedade e o estresse (Rocha et al., 2020).

Ao conhecer adequadamente o perfil sociodemográfico e epidemiológico de determinada população, torna-se possível propor maneiras de cuidado direcionadas. Essas ações assistenciais devem refletir sobre estratégias de acolhimento, estratificação e plano terapêutico no âmbito da atenção primária com articulação potente com o serviço especializado. O movimento da equipe em buscar lógicas de atendimento mais preciso para a população possibilita prevenção de sofrimento psíquico e físico, o que impacta diretamente a qualidade de vida dessas mulheres.

\section{Conclusão}

A DPC é uma condição com alta prevalência e que causa sofrimento físico e psíquico para as mulheres que a vivenciam. Conhecer o perfil da população atendida é fundamental para fazer uma assistência precisa. O papel da equipe interdisciplinar é fundamental e o diagnóstico preciso e precoce pode evitar diversos procedimentos desnecessários, além de abreviar a dor o sofrimento psíquico.

Os resultados do presente estudo corroboram com estudos internacionais e nacionais, contudo, trazem particularidades importantes relacionada ao perfil identificado. Essas diferenças devem ser cada vez mais estudas e articuladas para proposição de tratamentos eficazes destinados à determinada população, fazendo com que cada vez o plano terapêutico seja mais acurado, transversal e fundamentado no modelo biopsicossocial.

\section{Referências}

Ahangari, A. (2014). Prevalence of chronic pelvic pain among women: an updated review. Pain Physician Journal, 17(2), E141-E147. https://www.painphysicianjournal.com/linkout?issn=\&vol=17\&page=E141

Bercrombie, P. D. \& Learman L. A. (2012). Providing holistic care for women with chronic pelvic pain. Journal of obstetric, gynecologic, and neonatal nursing, 41(5), 668-679. https://doi.org/10.1111/j.1552-6909.2012.01403.x

Brilhante, A. V. M., Oliveira, L. A. F., Lourinho, L. A. \& Manso, A. G. (2019). Narrativas autobiográficas de mulheres com endometriose: que fenômenos permeiam os atrasos no diagnóstico? Physis: Revista de Saúde Coletiva, 29(3), e290307. https://doi.org/10.1590/s0103-73312019290307.

Bryant, C., Cockburn, R., Plante, A.-F. \& Chia, A. (2016). The psychological profile of women presenting to a multidisciplinary clinic for chronic pelvic pain: high levels of psychological dysfunction and implications for practice. Journal of Pain Research, 9, 1049-1056. https://doi.org/10.2147/JPR.S115065

Coelho, L. S., Brito, L. M., Chein, M. B., Mascarenhas, T. S., Costa, J. P., Nogueira, A. A. \& Poli Neto, O. B. (2014). Prevalence and conditions associated with chronic pelvic pain in women from São Luís, Brazil. Brazilian Journal of Medical and Biological Research, 47(9), 818-825. https://doi.org/10.1590/1414$431 \mathrm{X} 20143710$ 
Haggerty, C. L., Peipert, J. F., Weitzen, S., Hendrix, S. L., Holley, R. L., Nelson, D. B., Randall, H., Soper D. E., Wiesenfeld H. C. \& Ness R. B. (2005). Predictors of chronic pelvic pain in an urban population of women with symptoms and signs of pelvic inflammatory disease. Sexually transmitted diseases, 32(5), 293-299. https://doi.org/10.1097/01.olq.0000162361.69041.a5

Lin, F. C., Funk, J. T., Tiwari, H. A., Kalb, B. T., \& Twiss, C. O. (2018). Dynamic pelvic magnetic resonance imaging evaluation of pelvic organ prolapse compared to physical examination findings. Urology, 119, 49-54. https://doi.org/10.1016/j.urology.2018.05.031

Loduca, A. \& Samuelian, C. (2009). Avaliação psicológica: do convívio com dores crônicas à adesão ao tratamento na clínica de dor. In: Alves Neto, O. Dor, princípios e prática. Artmed.

Loduca, A., Müller, B. M., Amaral, R., Souza, A. C., Focosi, A. S., Samuelian, C., Yeng, L. T. \& Batista M. (2014). Chronic pain portrait: pain perception through the eyes of sufferers. Revista Dor, 15(1), 30-35. http://dx.doi.org/10.5935/1806-0013.20140008

Modesto, W. O. \& Bahamondes, L. (2011). Relationship between chronic pelvic pain and functional constipation in women of reproductive age. The Journal of reproductive medicine, 56(9-10), 425-430. https://pubmed.ncbi.nlm.nih.gov/22010528/

Rocha, J. N., Castro, L. E., Riccobene, V. M., Autran, M. S. M., Nogueira, L. A. C. \& Reis, F. J. J. (2020). Intensity of pain, disability and psychosocial factors in women with chronic pelvic pain: cross-sectional study. BrJP, 3(3), 239-244. http://dx.doi.org/10.5935/2595-0118.20200177

Romão, A. P. M. S., Gorayeb, R., Romão, G. S., Poli-Neto, O. B., Reis, F. J. C., Rosa-e-Silva, J. C. \& Nogueira, A. A. (2009). High levels of anxiety and depression have a negative effect on quality of life of women with chronic pelvic pain. The International Journal of Clinical Practice, 63(5), 707-711. https://doi.org/10.1111/j.1742-1241.2009.02034.x

Roth, R. S., Punch, M. R. \& Bachman, J. E. (2011). Patient beliefs about pain diagnosis in chronic pelvic pain: relation to pain experience, mood and disability. The Journal of reproductive medicine, 56(3-4), 123-129. https://pubmed.ncbi.nlm.nih.gov/21542529/

Silva, G. P., Nascimento, A. L., Michelazzo, D., Alves Junior, F. F., Rocha, M. G., Silva, J. C., Reis, F. J. C., Nogueira, A. A. \& Poli Neto, O. B. (2011). High prevalence of chronic pelvic pain in women in Ribeirão Preto, Brazil and direct association with abdominal surgery. Clinics, 66(8), 1307-1312. https://doi.org/10.1590/S1807-59322011000800001

Singh, P., Seo, Y., Ballou, S., Ludwig, A., Hirsch, W., Rangan, V., Iturrino, J., Lembo, A. \& Nee, J. W. (2019). Pelvic Floor Symptom Related Distress in Chronic Constipation Correlates With a Diagnosis of Irritable Bowel Syndrome With Constipation and Constipation Severity but Not Pelvic Floor Dyssynergia. Journal of neurogastroenterology and motility, 25(1), 129-136. https://doi.org/10.5056/jnm17139

Siqueira-Campos, V., Luz, R. A., Deus, J. M., Martinez, E. Z. \& Conde, D. M. (2019). Anxiety and depression in women with and without chronic pelvic pain: prevalence and associated factors. Journal of Pain Research, 12, 1223-1233. https://doi.org/10.2147/JPR.S195317

Till, S. R., As-Sanie, S. \& Schrepf, A. (2019). Psychology of Chronic Pelvic Pain: Prevalence, Neurobiological Vulnerabilities, and Treatment. Clinical obstetrics and gynecology, 62(1), 22-36. https://doi.org/10.1097/grf.0000000000000412

Tirlapur, S. A., Kuhrt, K., Chaliha, C., Ball, E., Meads, C. \& Khan, K. S. (2013). The 'evil twin syndrome' in chronic pelvic pain: a systematic review of prevalence studies of bladder pain syndrome and endometriosis. International journal of surgery, 11(3), 233-237. https://doi.org/10.1016/j.ijsu.2013.02.003

Williams, R. E., Hartmann, K. E., Sandler, R. S., Miller, W. C. \& Steege, J. F. (2004). Prevalence and characteristics of irritable bowel syndrome among women with chronic pelvic pain. Obstetrics and gynecology, 104(3), 452-458. https://doi.org/10.1097/01.aog.0000135275.63494.3d

Wozniak, S. (2016). Chronic pelvic pain. Annals of Agricultural and Environmental Medicine, 23(2), 223-226. https://doi.org/10.5604/12321966.1203880

Yosefm, A., Allaire, C., Williams, C., Ahmed, A. G., Al-Hussaini, T., Abdellah, M. S, Wong, F., Lisonkova, S. \& Yong, P. J. (2016). Multifactorial contributors to the severity of chronic pelvic pain in women. American journal of obstetrics and gynecology, 215(6), 760e1-e760.e14. https://doi.org/10.1016/j.ajog.2016.07.023 